PHYSICAI CHARACTERISTICS OF PARTICLE BEAMS

W. C. Roesch

Battelle

Pacific Northwest Laboratory

Richland, Washington

September 15, 1972

\begin{abstract}
This report was prepared $N$ O
sponsored by was prepared as an account of work

the United States nor the United Government. Neither

Commission, nor nor the United States Atomic Energy

their contractors, suy of their employees, nor any of

makes any warranty, contractors, or their employees,

legal liability or

pleteness

product or uny information, apparatus,

Drodurt nr procese disclosed, úi tepresents that its use

would not infringe privately owned rights.
\end{abstract}

Paper for oral presentation and for inclusion in the proceedings of the Conference on Particle Accelerators In Radiation Therapy, Los Alamos Scientific Laboratory, $2-4$ oct 72 .

* This paper is based on work performed under United States Atomic Energy Commission Contract AT(45-1)-1830. 


\section{DISCLAIMER}

This report was prepared as an account of work sponsored by an agency of the United States Government. Neither the United States Government nor any agency Thereof, nor any of their employees, makes any warranty, express or implied, or assumes any legal liability or responsibility for the accuracy, completeness, or usefulness of any information, apparatus, product, or process disclosed, or represents that its use would not infringe privately owned rights. Reference herein to any specific commercial product, process, or service by trade name, trademark, manufacturer, or otherwise does not necessarily constitute or imply its endorsement, recommendation, or favoring by the United States Government or any agency thereof. The views and opinions of authors expressed herein do not necessarily state or reflect those of the United States Government or any agency thereof. 


\section{DISCLAIMER}

Portions of this document may be illegible in electronic image products. Images are produced from the best available original document. 
PHYSICAL CHARACTERISTICS OF PARTICLE BEAMS*

W. C. Roesch

\author{
Battelle \\ Pacific Northwest Laboratory \\ Richland, Washington
}

Introduction

The ise of heavy charged particles (mesons, protons, alpha particles, and ions of even heavier atoms) for radiotherapy appears promising for at least two reasons: 1) heavy particles have depth-dose distributions that should be more advantageous than those of conventional radiations, and 2) heavy particles have high LET (linear energy transfer) along at least part of their paths and thus may benefit from the oxygen effect as discussed elsewhere at this meeting. This parer describes the physical characteristics of particle beams that are relevant to exploiting these two properties.

\title{
Absorbed Dose
}

"Absorbed dose" is a physical quantity. It is defined as the energy imparted to matter, per unit mass, by ionizing radiations.

\section{Depth-dose Curves}

Figure 1 shows a typical curve of absorbed dose versus depth of penetration of the beem into an absorbing material for monoenergetic, heavy, charged particles. Near the greatest depth the particles can reach, the curve rises to a maximum called the "Bragg peak." Prior to the peak there i.s a nearly flat region, often called the "plateau". Beyond the peak the dose drops to nearly zero; the dose at greater depths is due to secondary radiations generated by the particles.

Figure 2 contrasts the heavy-particle depth-dose distribution of Fig. 1 with those of ${ }^{60} \mathrm{Co}$ gamma rays (1) and $20 \mathrm{-MeV}$ electrons (2) and shows why the heavy particle irradiation should have an advantage over these more conventional radiations. The doses have been normalized to the dose at the Bragg peak. If the radiations were being used to treat a tumor at the depth of the peak, all to the same dose, the conventional radiations would. expose the normal tissues in front of and hehind the tumor to much higher doses than the heavy-particle beam would. Thus, the heavy-particle j.rradiation would produce less damage to normal tissues for the given tumor dose and should thus be easier on the patient -- or, for approximately the same doses to normal tissues, a higher dose could be delivered to the tumor.

The potential advantages of the heavy particles would be absent if the Bragg peak did not fall within the patient's body. Thus there is no need to consider particles whose ranges exceed body dimensions. T'able I gives examples of the energies of particles whose range is about $15 \mathrm{~cm}$ of tissue. (3) The table also lists the machine voltages necessary to produce the particles from fully ionized atoms. 
TABLE I

ENERGIES FOR 15-CM RANGE

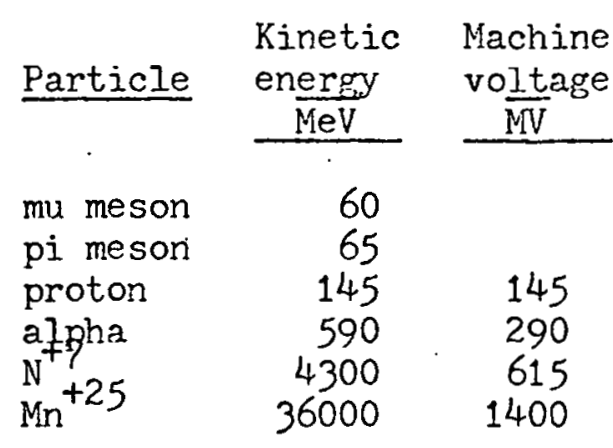

\section{The Bragg Peak}

The Bragg peak is the result of a complex interplay between the scattering, the slowing down, and the increase in the stopping power on slowing down undergone by the charged particles and their initial energy spread.

The dose produced by charged particles of a given energy is proportional to their stopping power, or LET . (c.f. 4) The dashed curve in Fig. 1 is proportional to the LET of particles whose range equals the difference between the range of the incident particles and the depth. It would be the depthdose curve, if all the particles lost energy at the same rate and suffered no scattering, for then all the particles would arrive at a given depoth with the same energy (and, hence, the same stopping power). However, because of repeated small-angle scattering, the particles do not move in perfectly straight lines; and, because of the stochastic nature of the energy-loss process, they do not lose energy at the same rate; thus, they arrive at a given depth with a distribution of energies. The dose is proportional to the average of the LET over this distribution. Actually, throughout, the Bragg-peak region, most of the particles are still of relatively high energy. Only a small fraction have the LET's characteristic of particles near the ends of their tracks. Thus the dose is much smaller than might have been expected from the dashed curve.

The Bragg peak of particles with a small spread in energy is broader and lower than that due to monoenergetic particles, because the initial distribution of energies further broadens the distribution of energies in the peak region. An energy spread of accelerated particles can result from limited energy resolution or from energy instability of the accelerator. Secondary particles such as pi mesons are produced with a wide spread of energies, and then a narrow band of energies is selected by magnetic analysis. The resulting spread contributes much of the width of their Bragg peak.

Bragg peaks are relatively narrow. For protons, their width at half maximum is about one-tenth of the depth at which they occur. Their absolute - ! width is thus on the order of one centimeter for therapeutically useful beams. The peak of the curve extends only over a millimeter or two. The degree of scattering and of energy straggling decrease with the mass of the particle; thus Bragg peaks are higher and narrower for heavier particles. For example, $\mathrm{N}^{+7}$ peaks are about one fourth as broad as those for protons at the same depth.

The narrowness of Bragg peaks makes them difficult to measure. The sensitive volume of the instrument must be much thinner than the peak, if the peak is to be portrayed accurately; otherwise the measurements will indicate a broader, lower peak than actually exists. 
Nuclear Reactions

Heavy particles of high energy produce nuclear reactions. This causes a reduction in the fluence of the particles, but it also generates fluences of secondary radiations, mostly other heavy particles, including neutrons. Unlike electron beams, bremsstrahlung ( $x$-ray) production by heavy particles is negligible. At very hjgh energies nuclear reactions strongly influence the shape of depth-dose curves -- they suppress the Bragg peak; however, at the relatively low energies of interest in therapy (see Table I) the effect is not very important. Negative pi mesons are an exception to this rule and are discussed separately below.

\section{Beam Width}

The dose distribution shown in Fig. 1 is for points along the axis of a broad beam. . If the beam is made narrower, there is little change until the radius of the beam becomes smaller than about one-tenth of the range of the particles. (5) Then, principally because scattering reduces the fluence of the particles near the end of the beam, the Bragg, peak begins to drop; the region of decreased dose extends further toward the surface of the medium as the beam becomes narrower. For very narrow beams, the top of the Bragg peak is lower than the plateau. Because of this beam-width effect, it is not possible to obtain a depth-dose advantage from the Bragg peak: for lesions smaller than about the width of the peak.

The actual width of the beam from an accelerator depends on the types of collimation and focusing employed. Beams as small as a fraction of a centimeter to as wide as several inches can be obtained. Scattering foils (e.g. thin sheets of tungsten or tantalum) placed in the beam help to make wide fields more uniform. Beam flattening may still be necessary. Use of shaped filters, as in x-ray or electron therapy, would shift the depth. of the Bragg peak and thus may be undesirable; it may be necessary to use a narrow but uniform field and scan by moving either the field or the patient.

\section{Radiation Quality}

The above considerations have treated the absorbed dose as the only quantity of interest, regardless of the type of radiation that produced the dose. This is not true; radiobiological evidence shows that some effects also depend on the type of radiation. In other words, radiations are of different 'qualities." In general, the difference in quality appears to be due to differences in the spatial distributions of energy transferred from charged particles to the medium along their tracks. For this reason, a.good first approximation to an index of quality of a charged-particle radiation is its LET. More powerful analyses can be made by the techniques of what is known as microdosimetry (6); however, although these techniques provided the distributions in LET used below, they will not be discussed further here because of the limited use that have been made of them so far.

Distributions in LET

At the surface of a medium irradiated by an accelerator beam, the charged particles are (nearly) monoenergetic; therefore, they all have (nearly) the same IET. 
As the particles penetrate the medium they lose energy and their LET increases. They do not all lose energy at the same rate (because of scattering and energy straggling), and they generate secondaries; thus, a spectrum of values: of the LET develops. The spectrum is roughly centered about the LET of particles of the mean energy at that depth.

In the region of the Bragg peak, significant numbers of particles near the ends of their tracks; they have IET's ten to one hundred times that of the incident particles. However, as noted above, the Bragg peak is not nearly this much higher than the plateal; therefore, the proportion of particles with the high LET's of nearly-stopped particles is fairly small.

On the downstream tail of the Bragg peak, the remaining incident particles must have very low energies, hence high LET. They will be few in number, however, and will be diluted with secondary radiations that may have a wide range of IET. The secondaries will constitute the entire spectrum beyond the Bragg peak.

Figures 3 and 4 illustrate these properties of the distributions in LET. The figures show the fraction of the dose delivered by charged particles whose LET's exceed the value of the abscissa. They were obtained (?) by the methods of microdosimetry. Consider the cuves labeled "nitrogen" in the two figures. The curve in Fig. 3 vas obtained near the surface of a medium irradiated by $3.9 \mathrm{GrV}$ nitrogen ions. Most of the dose comes from particles of near $19 \mathrm{keV} \mathrm{um}^{-1}$, the LET of the incident ions. The curve in Fig. 4 was obtained at the Bragg peak. A much wider range of LET's now contributes to the dose. Miost of the dose comes from particles with LET between 100 and $200 \mathrm{keV} \mathrm{um}{ }^{-1}$. The maxjmum LET for nitrogen ions is about $800 \mathrm{keV} \mathrm{um}^{-1}$, and very little of the dose comes from particles with that high an LET.

\section{Comparison of Radjations}

As will be discussed elsewhere in this meeting, the "quality" of charged particles changes rapidly as their LET exceeds something in the range 10 to $100 \mathrm{keV} \mathrm{um}^{-1}$. The radiation becomes more effective.

For comparison, Figs. 3 \& 4 show distributions for $14-\mathrm{MeV}$ neutrons in free space and at $12 \mathrm{~cm}$ in a phantom (8). Roughily $25 \%$ of the dose comes from the more effective LET's, but this is true at all depths so there is no differential sparing of overlying tissue. The corresponding distributions for ${ }^{60}$ would lie almost entirely to the left of $1 \mathrm{keV} \mathrm{um}^{-1}$, outside the area of the more effective LET's.

For the nitrogen beam, in the plateau region, the dose comes from particles that are largely below the region of the more effective LET's. At the Bragg peak, however, 80 to $90 \%$ or more of the dose comes from the more effective particles. Thus, the biological effectiveness of the Braggpeak region should be even greater than indicated by the peak in the absorbed dose.

Unfortunately, no distributions for lower-energy, ljghter particles. appear to have been obtained with which to compare the nitrogen distributions in Figs. 3.\& 4. For protons whose Bragg peak is at about the same depth as that for nitrogen, the surface distribution; corresponding to Fig. 3 . 
would be centered around an initial LET of about $0.7 \mathrm{keV} \mathrm{um}^{-1}$, well outside the range of the more effective values. The maximum IET of protons is 100 $\mathrm{keV} \mathrm{um}{ }^{-1}$. Therefore, the distribution at the Bragg peak, corresponding to Fig. 4, would lie so that a small part of its tail j.s above $100 \mathrm{keV} \mathrm{um}$. It is thus uncertain just what fraction of the Bragg peak dose would be more effective because of high enough IET. Clearly, however, the fraction would be less than in the case of the nitrogen beam. Thus, some ion heavier than the proton would be an optimum choice for therapeutic purposes; just what ion(s) would be best cannot be decided without experiments.

\section{Negative Pi Miesons}

Negative pi mesons merit a separate discussion, because, in addition to all of the considerations above, their depth-dose curves are modified by secondary radiations, particularly.radiations emitted as a consequence of nuclear reactions produced in the bragg-peak region. (9)

Negative pi mesons are especially productive of nuclear reactions, because when they have slowed down sufficiently (they are then at the Bragg peak) they are captured by atoms of the medium and occupy positions in the atoms similar to those of the orbital electrons. Being thus trapped in the vicinity of the nucleus, they are readily absorbed by the nucileus of all atoms except hydrogen; those captured by hydrogen generally exist long enough that they are transferred to and then absorbed in the nucleus of some heavier atom. Absorption in carbon, nitrogen, and oxygen, the most pabundant tissue elements, produces nuclear reactions in which gamma rays, neutrons, protons, alpha particles, and heavier ions such as those of lithium and carbon are emitted. About $100 \mathrm{NieV}$ of energy is shared among these secondary radiations. About $70 \%$ of the energy goes to neutrons and a few percent to gamma rays, which escape from the Bragg-peak region. The rest are absorbed within distances equal to their ranges and thus enhance the dose at the peak while broadening the peak slightly. Figure 5 shows a depth-dose curve calculated. (10) for a negative pi meson beam. The dashed line shows the Bragg peak that would exist without the contribution from the meson-capture reactions. The height and width of this latter curve are determined principally by the energy spread of the incident meson beam. The solid line gives the total dose resulting from the mesons and their secondaries.

Negative pi mesons are obtained as secondary particles from reactions in an accelerator target. Some electrons tertiary to the decay of uncharged mesons in the target accompany the negative pi mesons through the energy analyzing systern. Charged pi mesons decay into mu mesons and some of the latter will also accompany the pi mesons into the beam from the accelerator. These contaminant radiations will add to the doses shown in Fig. 5; they may add as much as 25 to $50 \%$ of the plateau dose. The contaminants are of longer range than the pi mesons so the added dose is nearly uniform through the depth-dose curve of the pi mesons and extends beyond the pi-meson Bragg peak.

The alpha particles and the heavier ions emitted in the meson-capture reactions have sufficiently high LET and are sufficiently abundant to affect the distribution of dose in IET at the Bragg peak Figure 3 shows: the distribution for a point on the plateau ( 11 ); essentially all the dose is from particles of LET less than $10 \mathrm{keV}$ um -1 , i.e. below the region of increased effectiveness. Figure 4 shows the distribution at the Bragg peak. 
The heavy particles have put 10 to $20 \%$ of the dose into the range of LET of increased effectiveness. Thus the effectiveness of the negative pi meson Bragg peak should be greater than indicated by relative absorbed doses. It may be possible to reduce the amount of contaminant radiations, which are low-IET, and thus increase the proportion of the more effective, high-IET radiaion.

\section{Field Modification}

The promising properties of particle beams, including the pi mesons, are asșociated with their Bragg peaks. The Bragg peaks are often narrow; they may be too narrow to cover a region a therapist may wish to treat. There are a number of ways of modifying accelerator radiation fields.

One method of obtaining a broader Bragg-peak region is to use higher energy particles. They will produce a broader peak but at a greater depth. The depth in the patient js then adjusted by adding absorbing material in front of the patient. This method also moves the surface of the patient further along the depth-dose curve, with a consequent increase in the entrance dose.

Figure 6 illustrates the combining of accelerator fields. Five depthdose distributions, indicated by solid lines, were added to give the combined field indicated by the line with dots. One of the component distributions is the one shom' in Fig. 1. The rest would be obtained from the sape accelerator beam by adding different amounts of absorbing material before the patient and exposing for shorter periods. The result is a peak 3 to 5 times wider than the original peak. It was obtained at the expense of a $60 \%$ increase in the plateau, or entrance, dose. Thus some of the advantage in depth-dose distribution is lost. However, it appears that, for reasonable treatment. volumes (several centimeters in diameter), the entrance dose will not exceed the tumor dose; thus, there will always be some advantage over photons and neutrons, which require larger entrance doses (see Fig. 1).

Combining fields will also combine their LET distributions. In the illustration, Fig. 6, the high-LE? radiation in the Bragg peaks of the smaller, displaced depth-dose distributions will be diluted with considerable low-LET radiation. Thus the LET distribution will not be uniform throughout the enlarged peak region; this neede further study.

Combining of fields can be done conveniently by oscillating an absorber (called.a "ridge filter" (12)) of suitable varied thickness in the accelerator beam. It could a.lso be done by variation of particle energy, if accelerator voltages were more easily changed. Combining by the conventional methods of multiple ports and of rotation can also be employed.

\section{Referencc}

1. Johns, H. E. (1969). X rays and teleisotope gamma rays, in Radiation Dosimetry, (F.H. Attix, W. C. Roesch, and E. Toshilin, eds.), second edition, vol. III, po. 1-50.

2. Radiation Dosimetry: Electrons with Initial Energies between 1 and 50 MeV, ICRU Report 21, International Commission on Radiation Units and Measurements, Washington, DC. 
3. Barkas, W. H. and Berger, M. J. (1964). Tables of Energy Losses and Ranges of Heavy Charged Particles, NASA-SP-3013, National Aeronautics and Space Administration, Washington, DC.

4. Roesch, W. C. and Attix, F. H. (1968). Basic concepts of dosimetry, in reference 1 , vol. I, pp. $1-41$.

5. Koehler, A. M. (1969). Quoted by M. R. Raju et al, in reference 1, pp. $151-199$.

6. Rossi, H. H. (1968). Microscopic energy distribution in irradiated matter, in reference 1 , vol. I, pp. 43-92.

7. Gross, W. (1972). Personal communication of the work of R. C. Rodgers, J. F. Dicello, and W. Gross.

8. Heintz, P. H., Robkin, M. A., Wootton, P. and Bichsel, H. (1971).

In-phantom microdosimetry with $14.6 \mathrm{MeV}$ neutrons, Health Physics 21 598-602.

9. Fowler, P. H. and Mayes, V. M. (1967). Proc. Phys. Soc. (Iondon) 22377.

10. Curtis, S. B. and Raju, M. R. (1968). A calculation of the physical

characteristics of negative pion beams -- energy-loss distribution and

Bragg curves, Radiation Research 34 239-255.

11. Lucas, A. C., Quam, W. M., and Raju, M. R. (1969). Microdosjmetry for a Pi Meson Beam, EG \& G, Inc., Goleta, California.

12. Karlsson, B. G. (1964). Methoden zur Berechnung und Erzielung Einiger fur die Tiefentherapie mit Hochenergetischen Protonen gunstiger Dosisverteilungen, Strahlentheravie 124481.

\section{Footnotes}

* This paper is based on work performed under United States Atomic Energy Commission Contract AT (45-1)-1830.

* No distinction is made in this paper between stopping power and linear energy transfer (LET). 


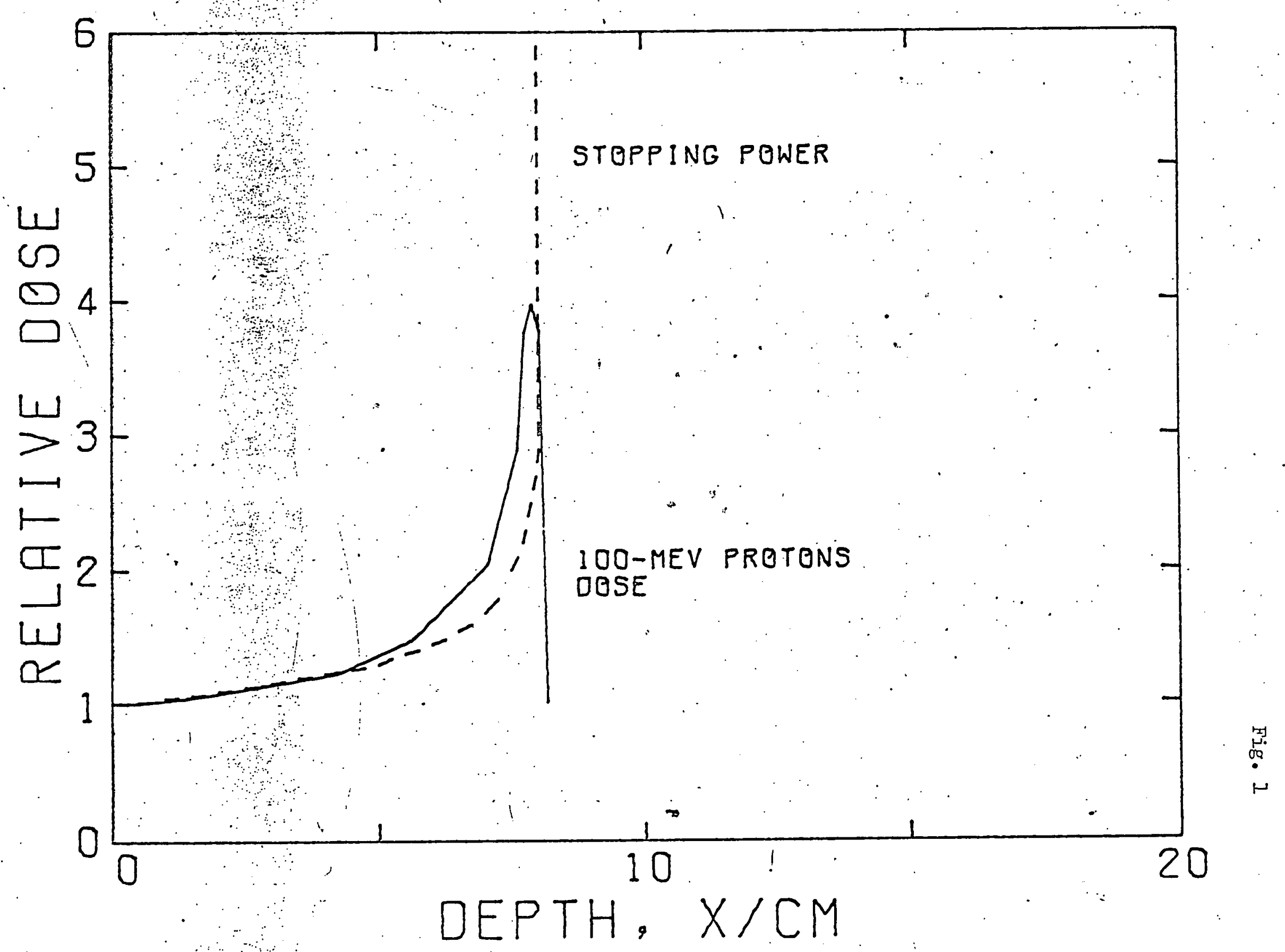

\% 


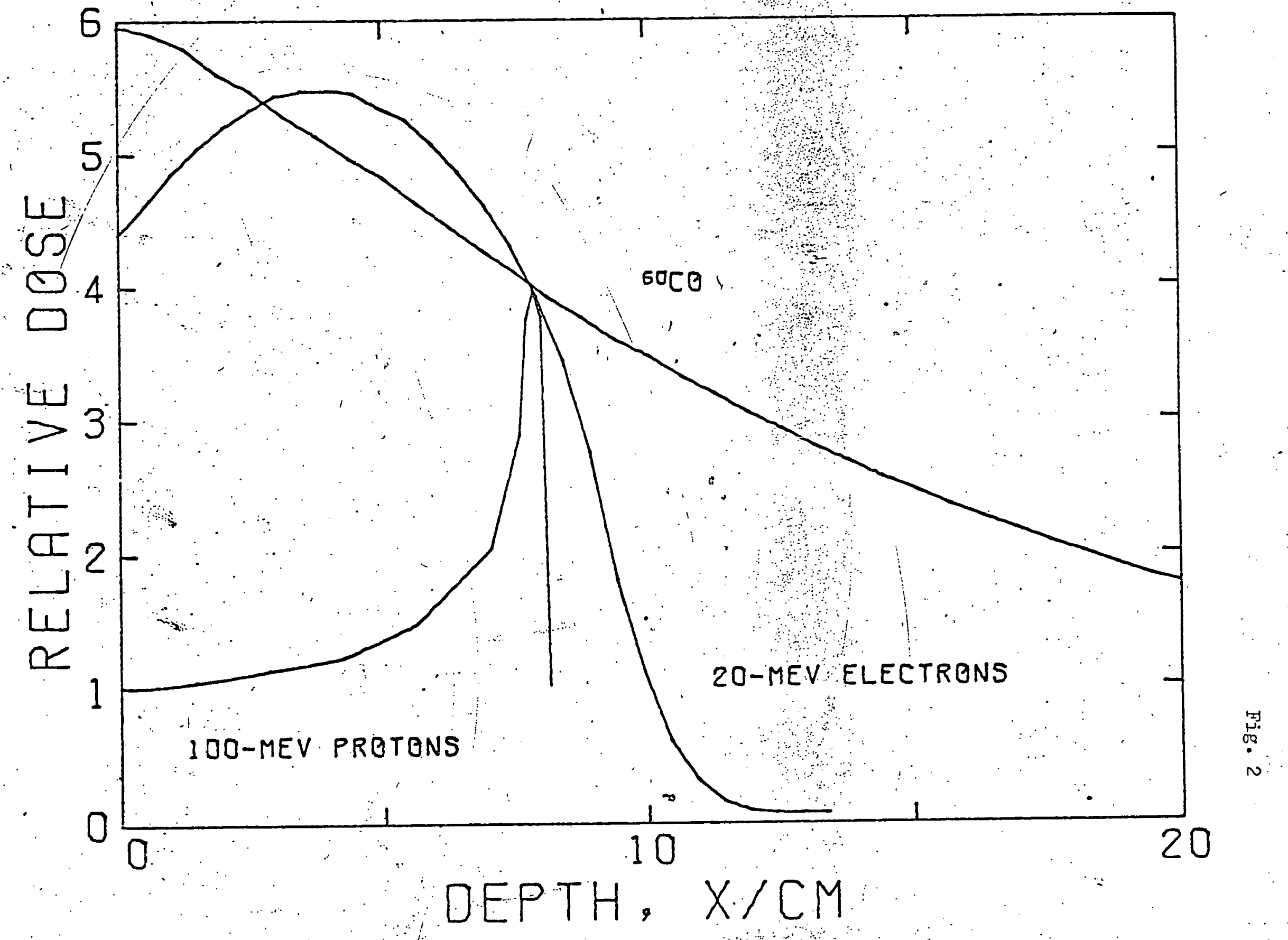



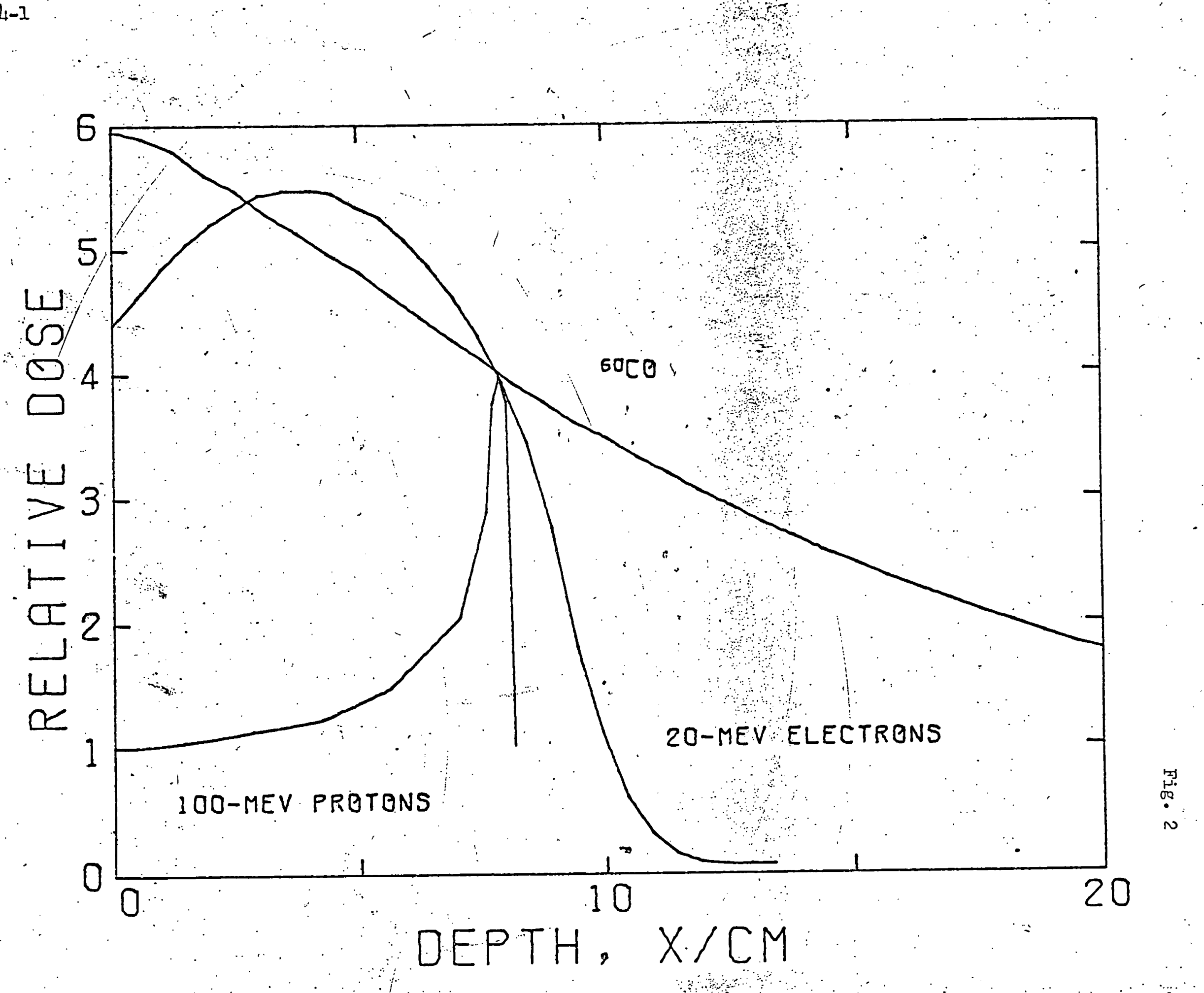


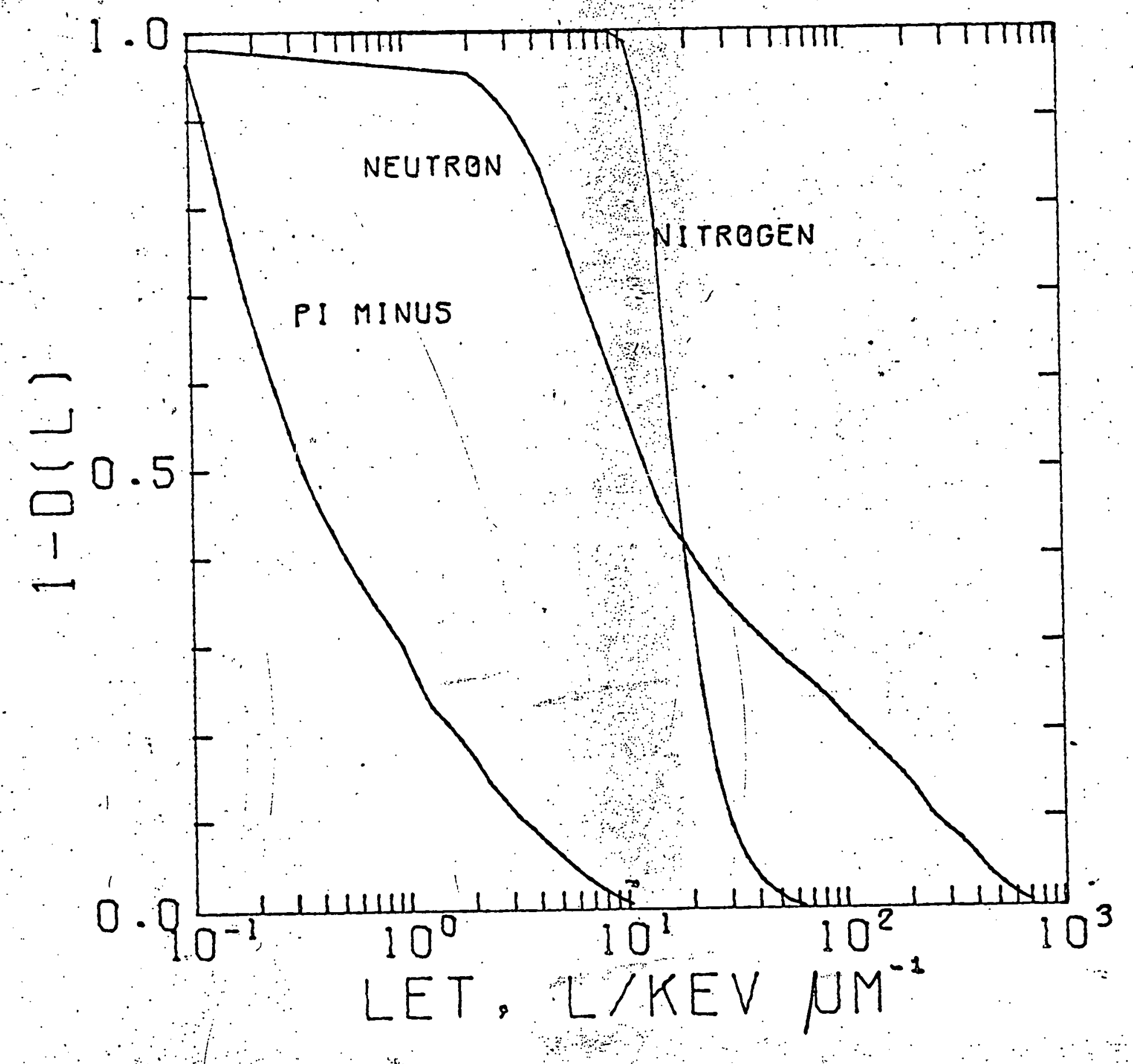




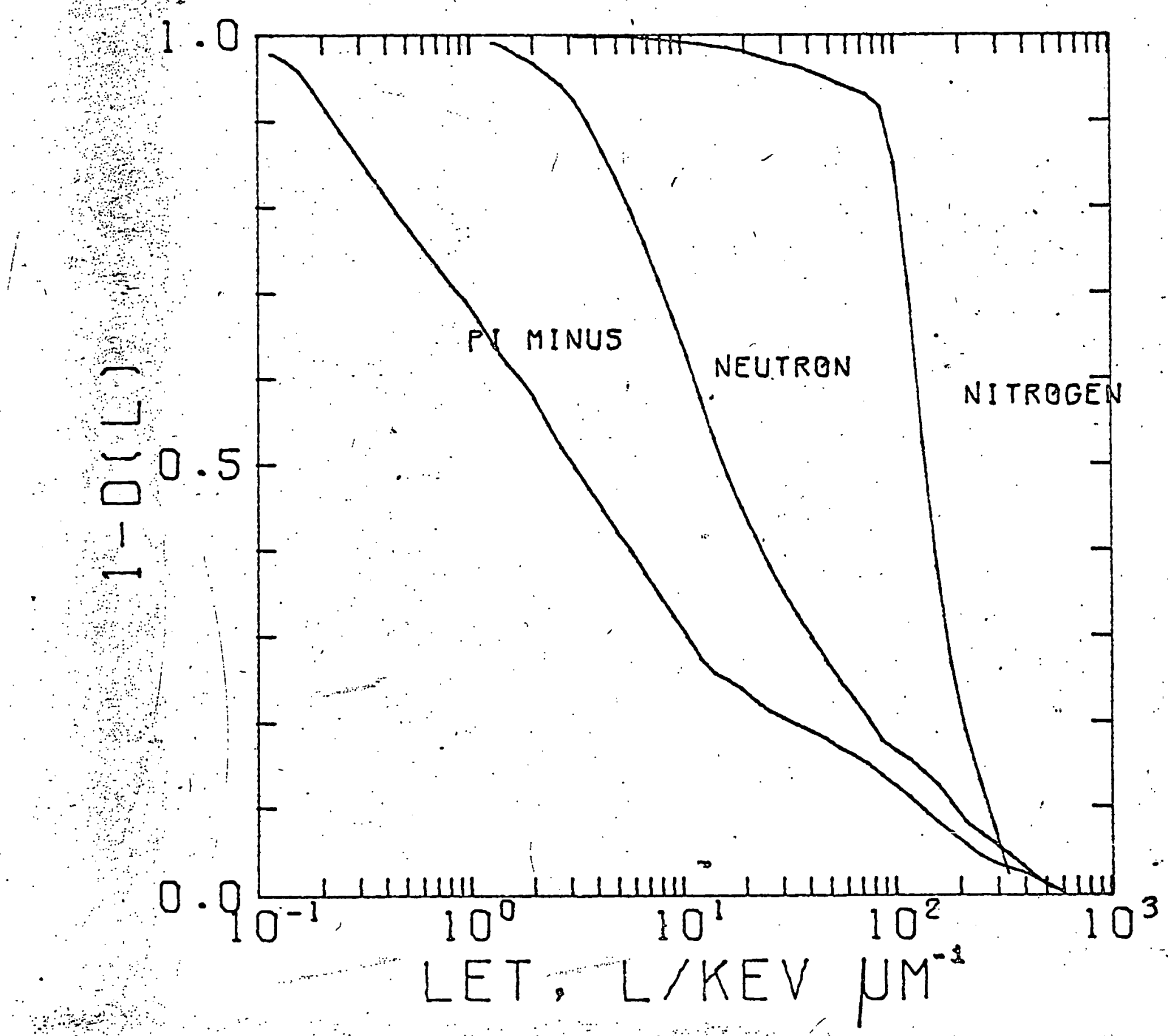


$72091 !-082 n$

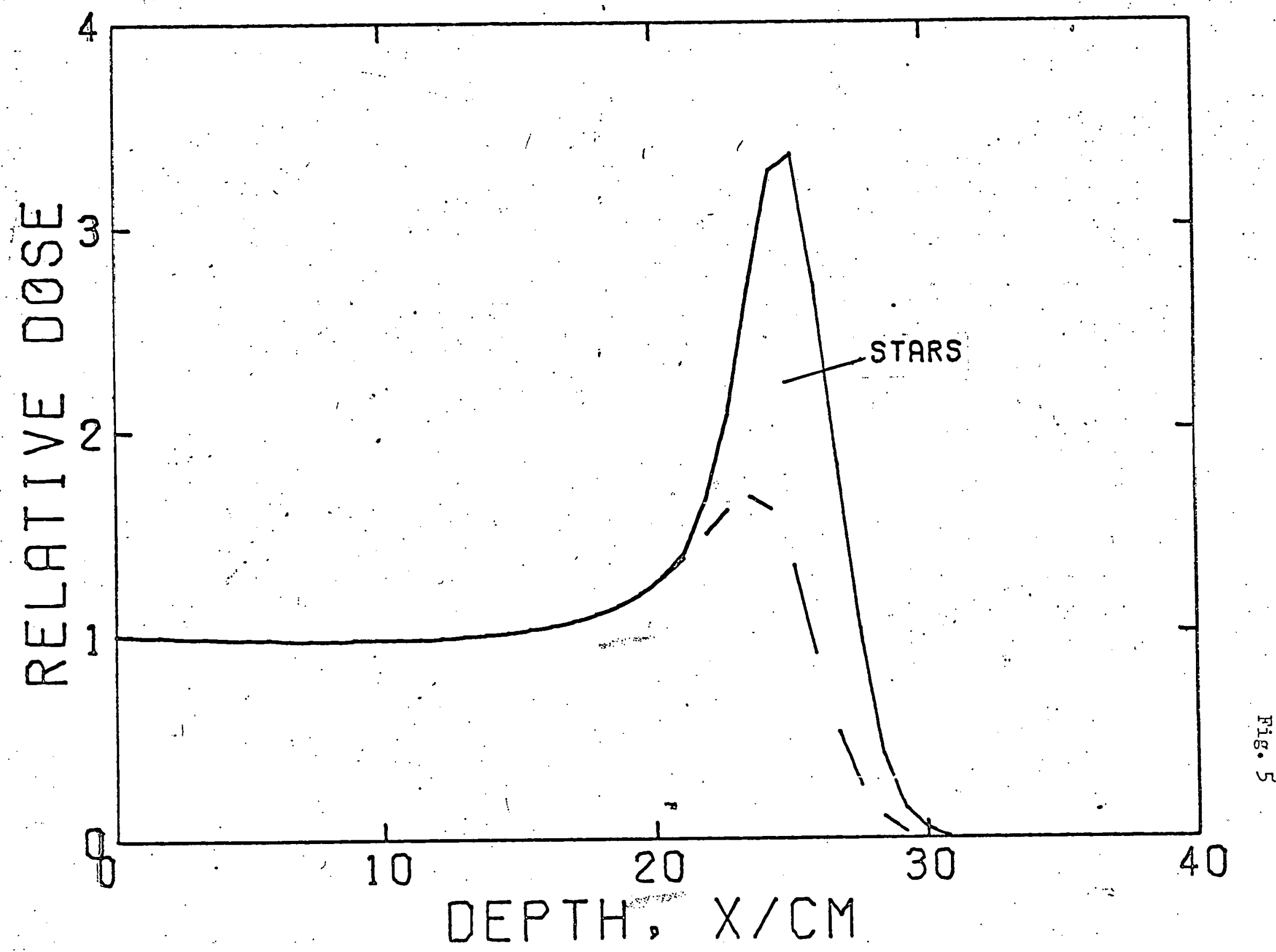




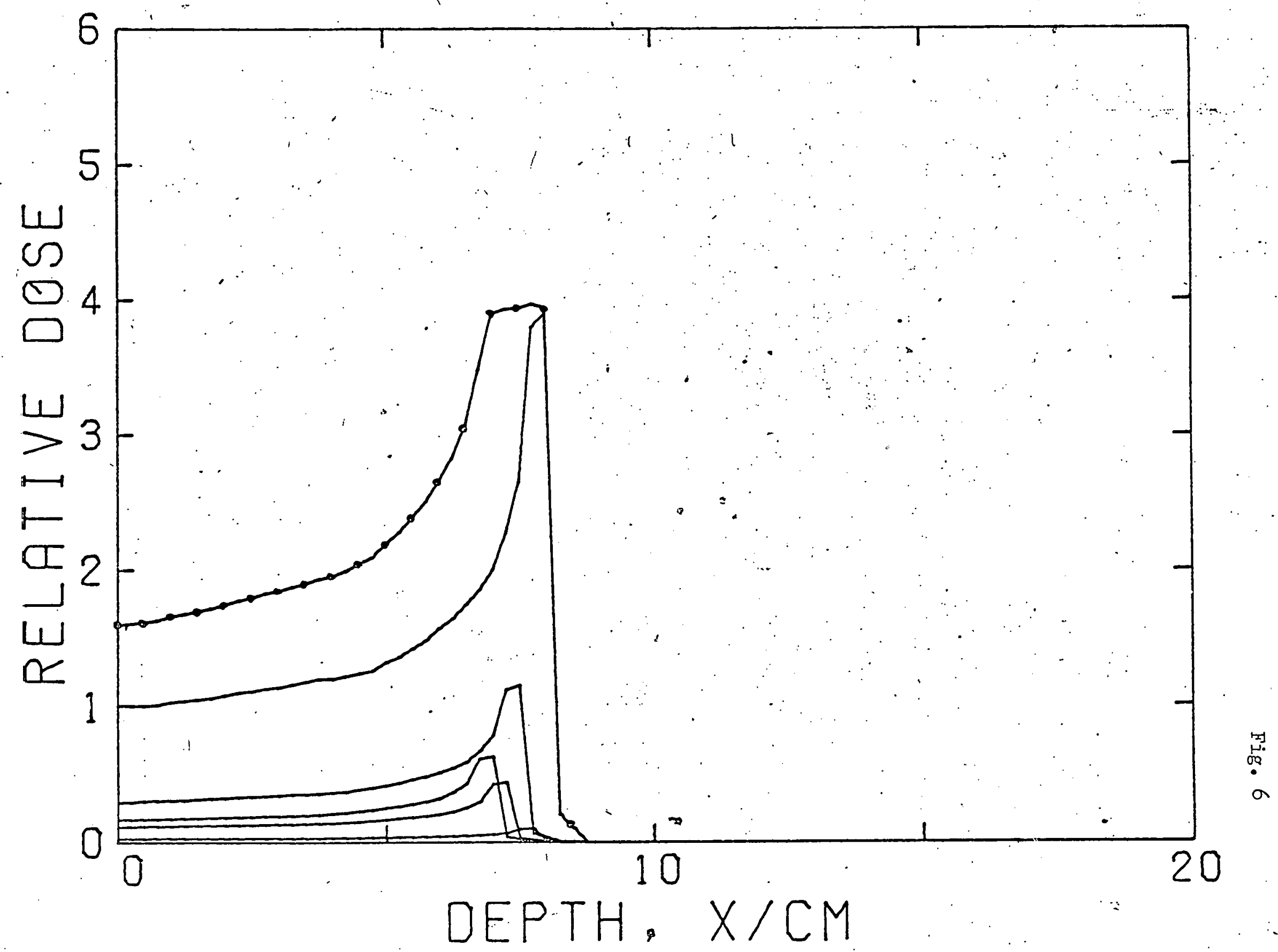

